\title{
Clumping of ejecta and accelerated cosmic rays in the evolution of type Ia SNRs
}

\author{
S. Orlando ${ }^{1}$, F. Bocchino ${ }^{1}$, M. Miceli ${ }^{1}$, O. Petruk ${ }^{2}$ and M. L. Pumo ${ }^{3}$ \\ ${ }^{1}$ INAF - Osservatorio Astronomico di Palermo, Piazza del Parlamento 1, 90134 Palermo, Italy \\ ${ }^{2}$ Inst. for Appl. Problems in Mechanics and Mathematics, Naukova St. 3-b Lviv 79060, Ukraine \\ ${ }^{3}$ INAF - Osservatorio Astronomico di Padova, Vicolo dell'Osservatorio 5, 35122 Padova, Italy
}

\begin{abstract}
We investigate the role played by initial clumping of ejecta and by efficient acceleration of cosmic rays (CRs) in determining the density structure of the post-shock region of a Type Ia supernova remnant (SNR) through detailed 3D MHD modeling. Our model describes the expansion of a SNR through a magnetized interstellar medium (ISM), including the initial clumping of ejecta and the effects on shock dynamics due to back-reaction of accelerated CRs. The model predictions are compared to the observations of SN 1006. We found that the back-reaction of accelerated CRs alone cannot reproduce the observed separation between the forward shock (FS) and the contact discontinuity (CD) unless the energy losses through CR acceleration and escape are very large and independent of the obliquity angle. On the contrary, the clumping of ejecta can naturally reproduce the observed small separation and the occurrence of protrusions observed in SN 1006, even without the need of accelerated CRs. We conclude that FS-CD separation is a probe of the ejecta structure at the time of explosion rather than a probe of the efficiency of CR acceleration in young SNRs.
\end{abstract}

Keywords. (magnetohydrodynamics:) MHD, (ISM:) cosmic rays, (ISM:) supernova remnants

\section{Introduction}

Current multi-dimensional models of supernova remnants (SNRs) predict an average distance $D$ between the contact discontinuity (CD) and the forward shock (FS) that is much larger than that observed in many young SNRs (e.g. SN1006) and cannot explain the high number of knots observed to protrude ahead of the shock. A possible cause invoked to explain these features has been the back-reaction of accelerated cosmic rays (CRs) at the FS. However, this mechanism cannot explain the evidence that the distance $D$ is lower and the occurrence of protrusions is higher than predicted even in regions where the local CRs acceleration efficiency is very low (e.g. Miceli et al. (2009)).

Nowadays, there is a growing consensus that density clumping of ejecta may be intrinsic at early phases of the remnant evolution (e.g. Maeda et al. (2010)). An important question is: can the ejecta clumping enhance the growth of hydrodynamic instabilities up to a level that allows them to reach and possibly overtake the FS? We investigate this issue by developing a 3D MHD model of SNR expanding through a magnetized medium, including consistently both the initial ejecta clumping and the effects on shock dynamics due to back-reaction of accelerated CRs (see Orlando et al. (2012) for a detailed description of the model and an extended discussion of the results).

\section{The MHD model and the results}

Our model describes the expansion of a SNR through a non-uniform magnetized medium and is described by the time-dependent MHD equations (Orlando et al. (2007), Orlando et al. (2012)). The cosmic ray back-reaction is taken into account by means of 
(A)

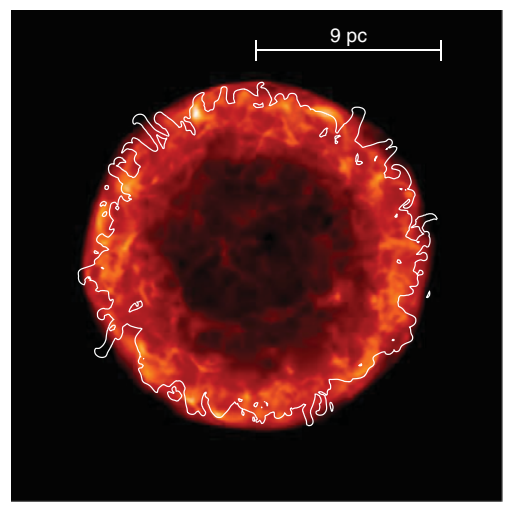

(B)

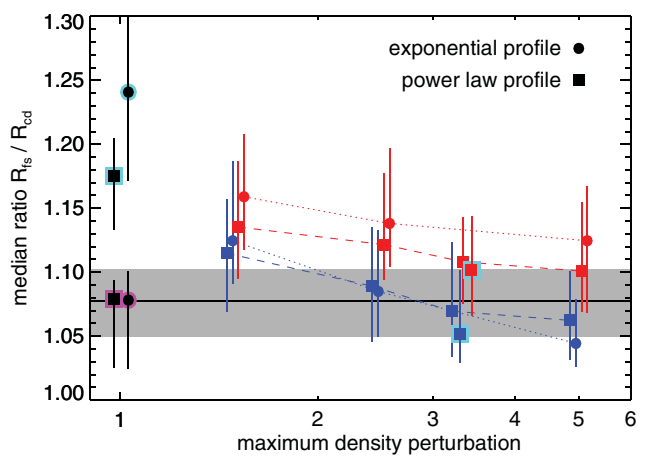

Figure 1. (A) 3D rendering of mass density for a model with an initial clumpy structure of the ejecta and no shock modification by CRs. The white contour encloses the original ejecta material. (B) Median values of the ratio of the forward shock radius to the contact discontinuity radius $R_{f s} / R_{c d}$ versus the maximum density perturbation for models either with (red and blue symbols for initial size of the clumps $\approx 1 \%$ and $\approx 2 \%$ of the remnant diameter, respectively) or without (black symbols) ejecta clumping, and for models including the back-reaction of accelerated CRs (symbols with a cyan or magenta halo). The grey region marks the range of values observed in SN1006 (Miceli et al. (2009)).

a space- and time-dependent effective adiabatic index $\gamma_{\text {eff }}$ by following the approach of Ferrand et al. (2010). The calculations are performed with the FLASH code (Fryxell et al. (2000)). As initial conditions we adopted parameters appropriate to describe the SNR SN1006. We also assume that the initial ejecta has a clumpy structure. The clumps have been modelled as per-cell random density perturbations (Orlando et al. (2012)).

We performed simulations either with or without the backreaction of accelerated CRs and either with or without the clumping of ejecta. For each simulation, we derived the median values of the ratio between the FS and the CD radii $R_{f s} / R_{c d}$ versus the maximum density perturbation as reported in Fig. 1 . We found that the back-reaction of accelerated CRs alone cannot reproduce the observations in SN1006 unless the CRs energy losses are extreme (i.e. $\gamma_{e f f} \approx 1.1$ ) and independent on the obliquity angle. An initial clumping of ejecta turns out to be a fundamental ingredient to reproduce the observed values of $R_{f s} / R_{c d}$ in SN1006 and its obliquity dependence. We conclude therefore that, in general, the separation between the FS and the CD is not a reliable diagnostic tool for studying the CR shock modification (see Orlando et al. (2012) for a full discussion of the results).

\section{Acknowledgements}

The software used in this work was in part developed by the DOE-supported ASC / Alliance Center for Astrophysical Thermonuclear Flashes at the University of Chicago.

\section{References}

Ferrand, G., Decourchelle, A., Ballet, J., Teyssier, R., \& Fraschetti, F. 2010, A\& A, 509, L10

Fryxell, B., et al. 2000, ApJS, 131, 273

Maeda, K., et al. 2010, Nature, 466, 82

Miceli, M., et al. 2009, Aछ A, 501, 239

Orlando, S., Bocchino, F., Reale, F., Peres, G., \& Petruk, O. 2007, A\&SA, 470, 927

Orlando, S., Bocchino, F., Miceli, M., Petruk, O., \& Pumo, M. L. 2012, ApJ, 749, 156 